Concept Paper

\title{
Metallization on FDM Parts Using the Chemical Deposition Technique
}

\section{Azhar Equbal * and Anoop kumar Sood}

Manufacturing Engineering, National Institute of Foundry and Forge Technology,

Ranchi 834003, India; E-Mail: anoopkumarsood@gmail.com

* Author to whom correspondence should be addressed; E-Mail: azhr_eqb106@yahoo.co.in;

Tel.: +91-0651-2292-067; Fax: +91-0651-2290-860.

Received: 7 May 2014; in revised form: 14 July 2014 / Accepted: 21 July 2014 /

Published: 5 August 2014

\begin{abstract}
Metallization of ABS (acrylonitrile-butadiene-styrene) parts has been studied on flat part surfaces. These parts are fabricated on an FDM (fused deposition modeling machine) using the layer-wise deposition principle using ABS as a part material. Electroless copper deposition on ABS parts was performed using two different surface preparation processes, namely ABS parts prepared using chromic acid for etching and ABS parts prepared using a solution mixture of sulphuric acid and hydrogen peroxide $\left(\mathrm{H}_{2} \mathrm{SO}_{4} / \mathrm{H}_{2} \mathrm{O}_{2}\right)$ for etching. After surface preparations using these routes, copper $(\mathrm{Cu})$ is deposited electrolessly using four different acidic baths. The acidic baths used are $5 \mathrm{wt} \%$ $\mathrm{CuSO}_{4}$ (copper sulfate) with $15 \mathrm{wt} \%$ of individual acids, namely $\mathrm{HF}$ (hydrofluoric acid), $\mathrm{H}_{2} \mathrm{SO}_{4}$ (sulphuric acid), $\mathrm{H}_{3} \mathrm{PO}_{4}$ (phosphoric acid) and $\mathrm{CH}_{3} \mathrm{COOH}$ (acetic acid). $\mathrm{Cu}$ deposition under different acidic baths used for both the routes is presented and compared based on their electrical performance, scanning electron microscopy (SEM) and energy dispersive X-ray spectrometry (EDS). The result shows that chromic acid etched samples show better electrical performance and $\mathrm{Cu}$ deposition in comparison to samples etched via $\mathrm{H}_{2} \mathrm{SO}_{4} / \mathrm{H}_{2} \mathrm{O}_{2}$.
\end{abstract}

Keywords: rapid prototyping; plastic; conductivity; surface preparation/conditioning; copper deposition 


\section{Introduction}

Plastic has many attractive properties, which include light weight, easy formability, reflectivity, abrasion resistance, electrical conductivity, etc. However, there are many areas in which, due to decorative or technological considerations, metallic properties are required or demanded [1]. It is in these applications that the possibility of metalizing plastics has opened the door to new uses for these materials. Metallization is a process in which a non-conductive material, such as plastic, is made conductive by providing a conductive layer on it. With metallization, the physical and mechanical properties of plastics, such as reflectivity, heat resistance, strength, etc., can be improved or may be changed as desired [2]. This has increased the possibility of utilizing the properties of metal and plastic in the same part material. As a result, plated plastic is used to address a variety of needs, from decorative finishes, such as chrome-plated automotive trim used in interior and exterior applications, automobile parts (such as dashes, dash boards, arm rests, vents, etc.) to functional requirements, such as controlling electro-magnetic emissions from electronic industries.

A variety of plastics, such as polythene, Teflon, polysulfone, polypropylene, acrylonitrilebutadiene-styrene (ABS), etc. can be metalized with different metals, like copper ( $\mathrm{Cu})$, zinc $(\mathrm{Zn})$, nickel $(\mathrm{Ni})$, gold $(\mathrm{Au})$, chromium $(\mathrm{Cr})$, silver $(\mathrm{Ag})$, etc. [3,4]. Having excellent electrical conductivity and being relatively inexpensive, $\mathrm{Cu}$ has been widely studied for metallization, and a variety of plastics have been plated. The different metallization process involves electroless plating, brushing a metal paint, the spray metal technique, dipping in a metal paint, sputtering and vacuum metallization [5-8]. Among these, electroless plating, because of its simplicity and cheapness, finds the widest application in the metallization of plastic. In this method, a thin metallic layer can be develop on the activated plastic surface by oxi-reduction reactions without the aid of an electric potential [9-12].

In the electroless procedure, deposition happens spontaneously on any surface without requiring any external electrical potential and processing. However, it is more difficult to control the process with regards to film thickness and uniformity. In addition to that, it is a multi-step procedure requiring a long deposition time and complex chemical solutions. Some of these chemicals are costly and environmentally hazardous. To overcome these limitations, a number of researchers proposed the elimination of multiple routes or the use of less costly and environment-friendly chemicals. For part fabrication, the fused deposition modeling machine (FDM) by Stratasys Inc. (Edina, MN, USA) is used. FDM is one of the rapid prototyping (RP) processes that fabricates the part on the layer-wise deposition principle directly from the CAD model of the part. Unlike other RP systems, which involve an array of lasers, powders and resins, this process uses heated thermoplastic filaments, which are extruded from the tip of a nozzle in a prescribed manner. The detail of this process has been given elsewhere in the literature $[13,14]$. The material used for part fabrication is ABS (ABS P400). It contains $90 \%-100 \%$ acrylonitrile-butadiene-styrene resin and may also contain mineral oil (0\%-2\%), tallow $(0 \%-2 \%)$ and wax $(0 \%-2 \%)$ [15]. The ABS part fabricated through FDM is better than flat ABS parts, as the parts produced through FDM have a higher surface roughness, which is advantageous for depositing the catalysts on the part during the metallization process. 


\section{Literature Review}

Various researchers have found that electroless plating can be efficiently done on plastic surfaces if a proper etchant solution is used prior to the plating process. Thus, etching or conditioning is an important step before carrying out electroless plating on plastics, as it is mainly responsible for the proper adhesion between the plastic substrate and the metallic layer [16]. Surface conditioning provides the substrate with cavities and, in some cases, a modified chemistry, which improves the wettability of the surface and sometimes renders a hydrophobic surface hydrophilic. The optimum conditioning time depends on the initial surface properties, which, in turn, are dependent on the composition and fabrication conditions used in production [17]. For the case of ABS plastics, the usual etching solutions are composed of chromic acid in aqueous sulfuric acid. To increase the feasibility of this process, a large number of researchers have contributed to the modification of the existing procedure.

Gui-xiang et al. [18] investigated the process of direct copper plating on plastics. They etched the substrate by $\mathrm{CrO}_{3} / \mathrm{H}_{2} \mathrm{SO}_{4}$ solution containing palladium $\left(\mathrm{Pd}^{2+}\right)$ ions, catalyzed by a $\mathrm{Pd} / \mathrm{Sn}$ colloid solution and accelerated in an alkaline solution containing copper ions. They found that $\mathrm{Pd} / \mathrm{Sn}$ colloid catalyst has good dispersivity and a uniform distribution of particles, which results in better catalysis and good activation. These all lead to the increased conductivity of plastic surface. Luan B. et al. [17] studied the chemical surface preparation for electroless plating of stereo lithography polymers. They conducted the contact angle analysis to assess the surface hydrophilicity, so as to optimize the preparation process. They have also used an etching solution of chromic acid and sulfuric acid. The applicability of this technology was verified by the subsequent metallization process. Teixeira et al. [19] carried out electroless copper deposition on plastic using etching solutions of sulfuric acid with hydrogen peroxide and/or nitric acid, replacing the conventional use of chromic acid to avoid its effect on the environment. They concluded that the plastic sheets can be conditioned with non-polluting solutions of $\mathrm{H}_{2} \mathrm{O}_{2}, \mathrm{HNO}_{3}$ and $\mathrm{H}_{2} \mathrm{SO}_{4}$ as an alternative to the $\mathrm{Cr}(\mathrm{VI}) / \mathrm{H}_{2} \mathrm{SO}_{4}$ solutions that are conventionally used in industry. Shu et al. [20] investigated the environment-friendly palladium free surface activation techniques. Fritz et al. [21] carried out electroless deposition of copper on organic and inorganic substrates using a Sn/Ag catalyst. They investigated the electroless deposition of copper and silver on epoxy and silicon dioxide-based substrates. In their experiment, they found that a cost-efficient $\mathrm{Sn} / \mathrm{Ag}$ catalyst can be used as a replacement for the $\mathrm{Sn} / \mathrm{Pd}$ catalyst currently used in board technology. $\mathrm{Wu}$ et al. [22] studied the structure and properties of electrolessly deposited copper on different substrates under different plating conditions and demonstrated their success of applying molecular dynamics simulation to the investigation of the growing process of copper crystals. Ono et al. [23] investigated a direct copper plating system in which tin is removed from the palladium/tin catalyst particles by immersion in an accelerating solution containing copper ions. It is believed that the remarkable promotion effect of copper ions added in the accelerating solution on the lateral propagation speed by a modified mechanism of stepwise propagation through dispersed copper particle seeds. Naruskevicius et al. [24] investigated the use of cobalt (Co)-based surface activator for electroless copper deposition. They investigated the activation procedures of the surface by using the colloidal solution of cobalt compounds with the aim of using it as a palladium free activation solution prior to electroless copper deposition. Based on the literature survey in this study, chromic acid is compared with a mixture of sulfuric acid and hydrogen peroxide $\left(\mathrm{H}_{2} \mathrm{SO}_{4} / \mathrm{H}_{2} \mathrm{O}_{2}\right)$ for their performance. 
$\mathrm{H}_{2} \mathrm{SO}_{4} / \mathrm{H}_{2} \mathrm{O}_{2}$ is selected, as it is cheaper than tin and silver, which has been investigated by many researchers as a replacement for chromic acid.

\section{Methodology}

Two different routes were implemented for preparing the ABS surface for electroless copper deposition, as depicted in Figure 1. All reagents used in the present study were analytically pure reagent grade.

Figure 1. Different routes for the metallization of acrylonitrile-butadiene-styrene (ABS) plastic.

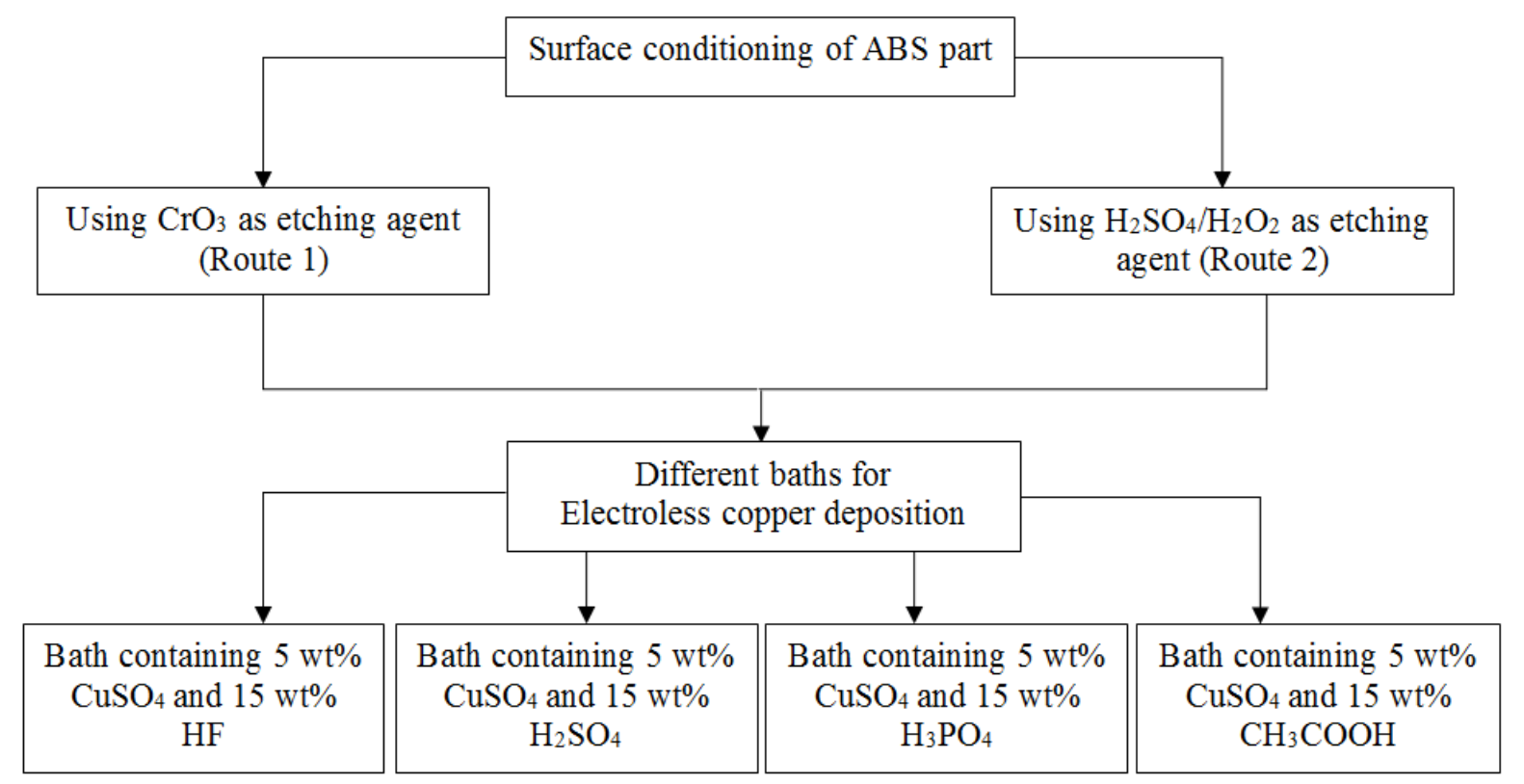

In Route 1, chromic acid $\left(\mathrm{CrO}_{3}\right)$ was used for etching the sample surface. In Route 2, all of the procedures of surface conditioning used in Route 1 were used, except for etching, where sulfuric acid $\left(\mathrm{H}_{2} \mathrm{SO}_{4}\right)$ and hydrogen peroxide $\left(\mathrm{H}_{2} \mathrm{O}_{2}\right)$ were used instead of chromic acid. After surface conditioning, these samples were dipped in four different acidic baths. The different acidic bath used for the electroless deposition of copper consist of $5 \mathrm{wt} \%$ of copper sulfate $\left(\mathrm{CuSO}_{4}\right)$ and $15 \mathrm{wt} \%$ of individual acids, namely hydrofluoric acid (HF), sulfuric acid $\left(\mathrm{H}_{2} \mathrm{SO}_{4}\right)$, phosphoric acid $\left(\mathrm{H}_{3} \mathrm{PO}_{4}\right)$ and acetic acid $\left(\mathrm{CH}_{3} \mathrm{COOH}\right)$. The detail of the different routes adopted for achieving the successful deposition of copper film on ABS plastic parts is presented as follows:

Route 1:

This is a chemical treatment route consisting of multi-step operations. The chemical reagent for etching in this process consists of chromic acid. The various steps are:

Step 1, Cleaning: The ABS sample was first cleaned by brushing with pumice powder and then scouring with sand paper to remove oil, dirt, grease, etc., and also to develop micro-roughness for increasing the surface area.

Step 2, Etching: In this step, the pre-cleaned ABS parts were dipped in an aqueous solution containing chromic acid (600 g/L), sulfuric acid $(150 \mathrm{~mL} / \mathrm{L})$ and deionized water. The solution was prepared by 
adding chromic acid and sulfuric acid slowly into stirring deionized water. After that, the temperature of the solution was raised to $60{ }^{\circ} \mathrm{C}$ and maintained at that temperature. The ABS samples were immersed in the bath for 10-15 min. The samples were then taken out and washed 2-3 times carefully.

Step 3, Neutralization: In this stage, the residual amount of chromium that remains in the ABS surface was removed with sodium sulfite as a reducing agent, so as to prevent its inhibition in further steps. It was believed that even trace amounts of chromium may completely inhibit electroless deposition. The ABS parts were dipped in solution of $10 \mathrm{~g} / \mathrm{L}$ of sodium sulfite at $25{ }^{\circ} \mathrm{C}$ for about 2 min and, finally, washed with water.

Step 4, Activation: The conditioned surface was contacted with an activator or catalysts consisting of a colloidal suspension of palladium/tin $(\mathrm{Pd} / \mathrm{Sn})$ catalyst powder $[25,26]$. The catalyst particles get deposited in the micro-cavities formed in the surface during the conditioning process. It is desirable not to put too much activator on the work being processed and to avoid too long of an immersion time. In the present study, samples were immersed at $40{ }^{\circ} \mathrm{C}$ for $7 \mathrm{~min}$ and, finally, washed with water.

Step 5, Acceleration: The accelerator dissolves excess $\mathrm{Sn}$ and removes it from the surface to expose the adsorbed $\mathrm{Pd}$. The solution contains a mixture of $30 \mathrm{~g} / \mathrm{L}$ sodium hydroxide $(\mathrm{NaOH}), 3 \mathrm{~g} / \mathrm{L}$ copper sulfate $\left(\mathrm{CuSO}_{4}\right)$ and $15 \mathrm{~g} / \mathrm{L}$ ethylenediaminetetraacetic acid disodium (EDTANa $)$. This was done at $55{ }^{\circ} \mathrm{C}$ for about $7 \mathrm{~min}$. The samples were finally washed with water and then dipped in individual acidic baths for different times.

\section{Route 2:}

This route differs with respect to Route 1 only in the etching stage. All other steps used in this route are the same as in Route 1 with the same constituents and compositions. For etching purposes, chromic acid was replaced with a solution of sulfuric acid $\left(\mathrm{H}_{2} \mathrm{SO}_{4}\right)$ and hydrogen peroxide $\left(\mathrm{H}_{2} \mathrm{O}_{2}\right)$. The ABS parts were immersed in the etching solution of $192 \mathrm{~mL}$ hydrogen peroxide $\left(\mathrm{H}_{2} \mathrm{O}_{2}\right), 160 \mathrm{~mL}$ sulfuric acid $\left(\mathrm{H}_{2} \mathrm{SO}_{4}\right)$ and $448 \mathrm{~mL}$ deionized water $\left(\mathrm{H}_{2} \mathrm{O}\right)$ maintained at room temperature for about $10 \mathrm{~min}$ [19]. After that, the parts were finally washed with water.

\subsection{Electrical Performance Measurement}

A digital multi-meter (VOLTCRAFT M-3850) was used to measure the resistance of the metalized ABS parts at different points on the surface. The average resistance $(\bar{R})$ value together with the standard deviation $(\sigma)$ was calculated. The deposition process, which yields the lower average resistance value along with a lower standard deviation among all of the listed methods, was considered better.

\subsection{Adhesion Assessment}

Adhesion assessment of the Al-seeded coated ABS samples was performed after electroless copper deposition in different baths by following the standard ASTM test method, ASTM D 3359-02 [27]. All the different samples from the $\mathrm{HF}, \mathrm{H}_{2} \mathrm{SO}_{4}, \mathrm{H}_{3} \mathrm{PO}_{4}$ and $\mathrm{CH}_{3} \mathrm{COOH}$ baths were tested. The surface is first cleaned carefully, and a thin grid of lines about $1 \mathrm{~mm}$ apart was cut over a surface. The cuts were of a sufficient depth to reach the ABS surface. A piece of adhesive tape was stuck onto the surface, and a $25 \mathrm{~kg}$ weight was placed on it for $5 \mathrm{~min}$. After that, the tape was taken off, and the resulting surface was examined as per ASTM D 3359-02. 


\subsection{SEM/EDS Characterization}

A ZEISS EVO- MA10 SEM scanning electron microscope (SEM, Carl Zeiss, Oberkochen, Germany) coupled with an energy dispersive X-ray spectrometer (EDS, Zeiss, Oberkochen, Germany) was used to examine the appearance and elemental composition of the $\mathrm{Cu}$-deposited ABS surfaces.

\section{Result and Discussion}

\subsection{Electrical Performance of Copper Deposited ABS Parts}

Tables 1 and 2 show the electrical performance of samples whose surfaces were prepared using Route 1 and Route 2, respectively. These readings are taken at room temperature after $1 \mathrm{~h}, 24 \mathrm{~h}$ and $48 \mathrm{~h}$ of deposition time. It is important to mention that conductivity is not obtained at all of the measured points; hence, the average resistance $(\bar{R})$ and standard deviation $(\sigma)$ have been calculated only for those points where the resistance value is obtained. Conductivity is obtained only for the HF and $\mathrm{H}_{2} \mathrm{SO}_{4}$ bath and not for the $\mathrm{H}_{3} \mathrm{PO}_{4}$ and $\mathrm{CH}_{3} \mathrm{COOH}$ bath for samples prepared through Route 1 (Table 1). For samples prepared through Route 2, except the $\mathrm{CH}_{3} \mathrm{COOH}$ bath, all of the acidic baths make the sample conductive (Table 2). For both of the individual routes, the best conductivity is obtained in the $\mathrm{H}_{2} \mathrm{SO}_{4}$ bath after $48 \mathrm{~h}$ of deposition time. However, it is to be noted that the resistance value through these routes is in $\mathrm{M} \Omega$ ).

Table 1. Electrical performance of samples prepared from Route 1 after electroless $\mathrm{Cu}$ deposition from different acidic baths.

\begin{tabular}{|c|c|c|c|c|c|c|c|c|}
\hline \multirow{3}{*}{ Deposition Time (h) } & \multicolumn{8}{|c|}{ Acidic Bath } \\
\hline & \multicolumn{2}{|l|}{ HF } & \multicolumn{2}{|c|}{$\mathrm{H}_{2} \mathrm{SO}_{4}$} & \multicolumn{2}{|l|}{$\mathrm{H}_{3} \mathrm{PO}_{4}$} & \multicolumn{2}{|c|}{$\mathrm{CH}_{3} \mathrm{COOH}$} \\
\hline & $\bar{R}$ (M $\Omega)$ & $\boldsymbol{\sigma}$ & $\bar{R}$ (M $\Omega)$ & $\boldsymbol{\sigma}$ & $\bar{R}$ (M $\Omega)$ & $\sigma$ & $\bar{R}(\mathbf{M} \Omega)$ & $\sigma$ \\
\hline 1 & - & - & - & - & - & - & - & - \\
\hline 24 & 0.12 & 0.02 & 0.11 & 0.03 & - & - & - & - \\
\hline 48 & 0.11 & 0.01 & 0.09 & 0.01 & - & - & - & - \\
\hline
\end{tabular}

Table 2. Electrical performance of samples prepared from Route 2 after electroless $\mathrm{Cu}$ deposition from different acidic baths.

\begin{tabular}{|c|c|c|c|c|c|c|c|c|}
\hline \multirow{3}{*}{ Deposition Time (h) } & \multicolumn{8}{|c|}{ Acidic Bath } \\
\hline & \multicolumn{2}{|l|}{ HF } & \multicolumn{2}{|c|}{$\mathrm{H}_{2} \mathrm{SO}_{4}$} & \multicolumn{2}{|c|}{$\mathrm{H}_{3} \mathrm{PO}_{4}$} & \multicolumn{2}{|c|}{$\mathrm{CH}_{3} \mathrm{COOH}$} \\
\hline & $\bar{R}$ (M $\mathbf{\Omega})$ & $\boldsymbol{\sigma}$ & $\bar{R}(\mathbf{M} \Omega)$ & $\boldsymbol{\sigma}$ & $\bar{R}$ (M $\Omega)$ & $\boldsymbol{\sigma}$ & 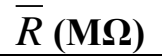 & $\sigma$ \\
\hline 1 & - & - & - & - & - & - & - & - \\
\hline 24 & 4.87 & 1.01 & 2.15 & 0.61 & 3.07 & 0.52 & - & - \\
\hline 48 & 4.15 & 0.56 & 2.10 & 0.39 & 2.95 & 0.55 & - & - \\
\hline
\end{tabular}

To understand the difference in conductivity in each route and different acidic baths, the growth and distribution of $\mathrm{Cu}$ on different samples were studied using SEM (scanning electron microscope) images of all of the treated parts. The EDS (energy dispersive spectrometer) of $\mathrm{Cu}$-deposited ABS 
samples were also performed for typical EDS spectra. As good conductivity was obtained after a 48-h deposition time, only SEM and EDS data at this particular time are presented in the next subsection.

\subsection{SEM and EDS Characterization of Samples of Route 1}

SEM and EDS image for the sample dipped in HF bath at room temperature for $48 \mathrm{~h}$ of deposition time is shown in the Figure 2. Cu deposition is not clearly visible in SEM. However, the EDS spectra show that the $\mathrm{Cu}$ peak is present in addition to some other elements. The presence of the copper peak and the height of copper peak dictate the presence of copper with weight percentage. The composition of $\mathrm{Cu}$ among these elements is $0.32 \%$. The images for the sample dipped in the $\mathrm{H}_{2} \mathrm{SO}_{4}$ bath is presented in Figure 3. It consists of the SEM image and EDS spectra of the electroless deposited sample. Large $\mathrm{Cu}$ crystals can be clearly viewed in the SEM image. Elemental analysis shows $88.83 \%$ $\mathrm{Cu}$ is there in addition to the other element.

Figure 2. (a) SEM image and (b) EDS spectra of Cu electrolessly deposited on the sample whose surface is prepared through Route 1 in the HF bath.

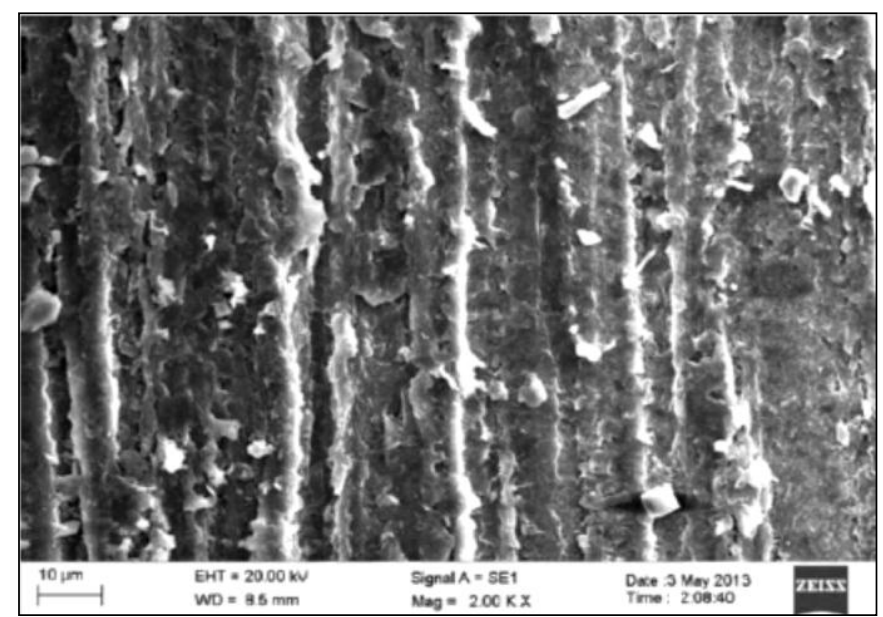

(a)

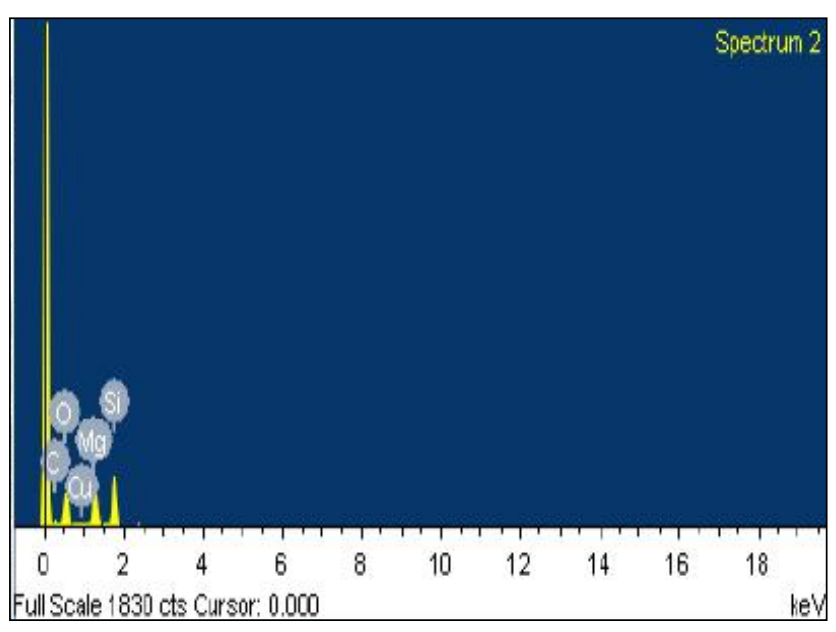

(b)

Figure 3. (a) SEM image and (b) EDS spectra of Cu electrolessly deposited on the sample whose surface is prepared through Route 1 in the $\mathrm{H}_{2} \mathrm{SO}_{4}$ bath.

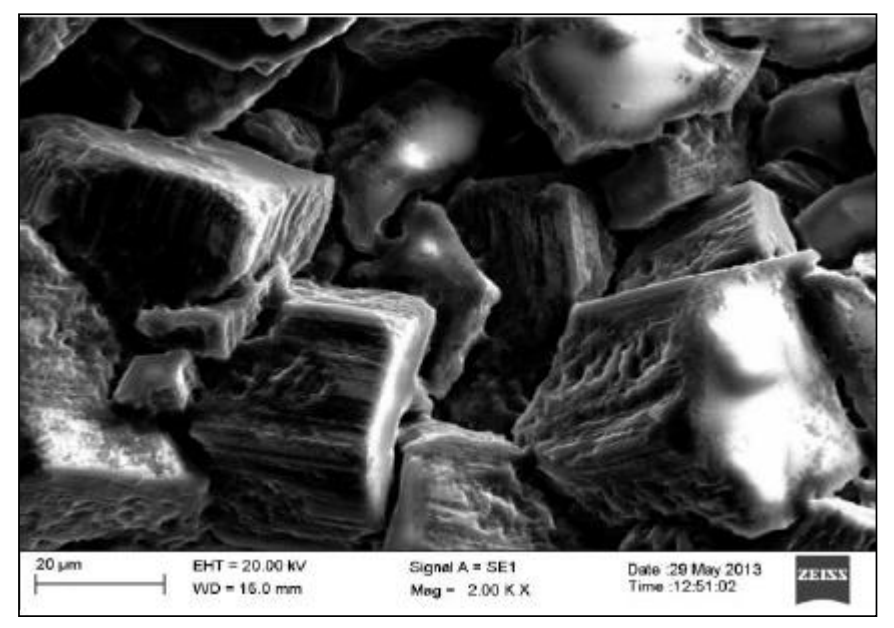

(a)

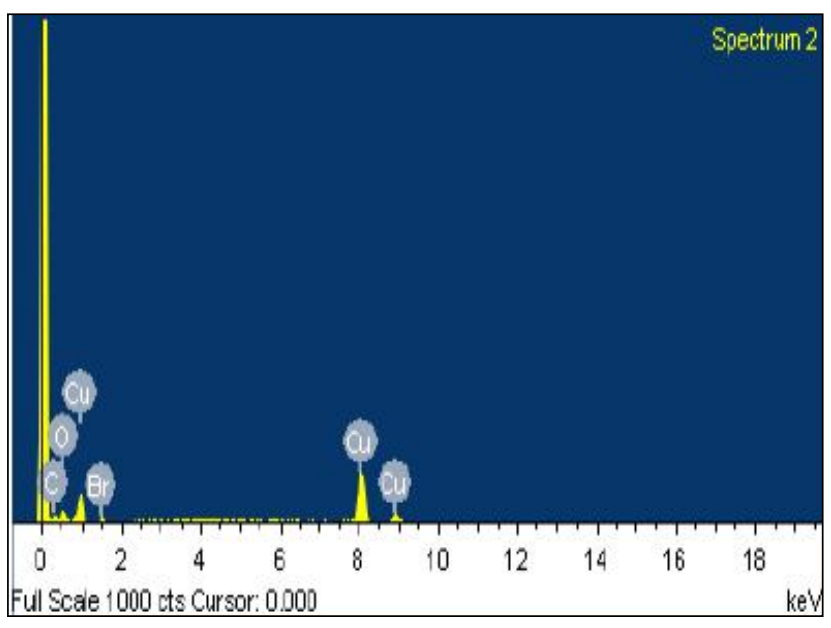

(b) 
Figure 4 represents the SEM image and EDS spectra of the sample deposited in the $\mathrm{H}_{3} \mathrm{PO}_{4}$ bath for $48 \mathrm{~h}$ of deposition time. The SEM image has a very poor appearance with no visible copper. EDS spectra also make it clear that no copper is present. Figure 5 shows the SEM and EDS image for the sample dipped in the $\mathrm{CH}_{3} \mathrm{COOH}$ bath for $48 \mathrm{~h}$ of deposition time. The SEM image is very poor, with no $\mathrm{Cu}$ crystals at all. The EDS spectra of the sample are also shown in the figure.

Figure 4. (a) SEM image and (b) EDS spectra of Cu electrolessly deposited on the sample whose surface is prepared through Route 1 in the $\mathrm{H}_{3} \mathrm{PO}_{4}$ bath.

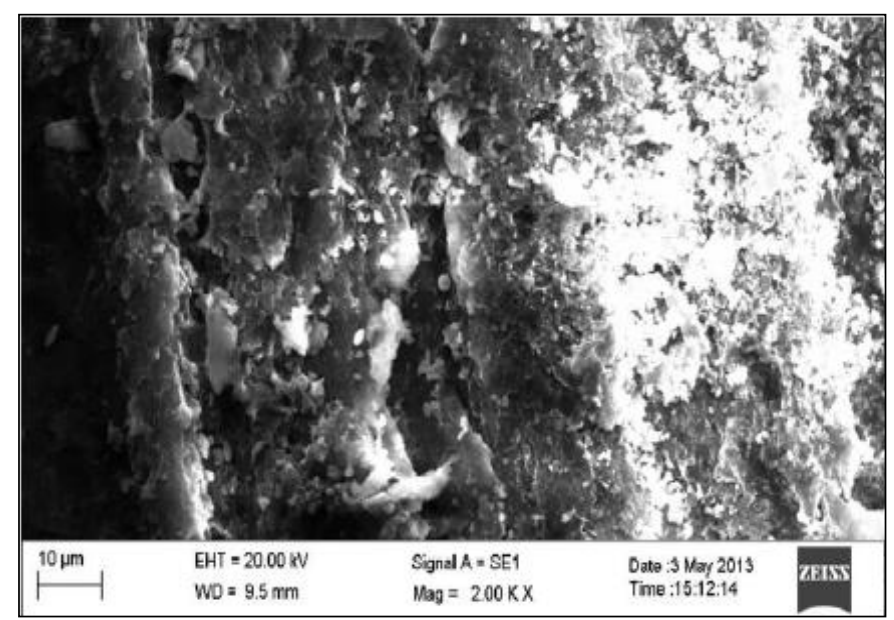

(a)

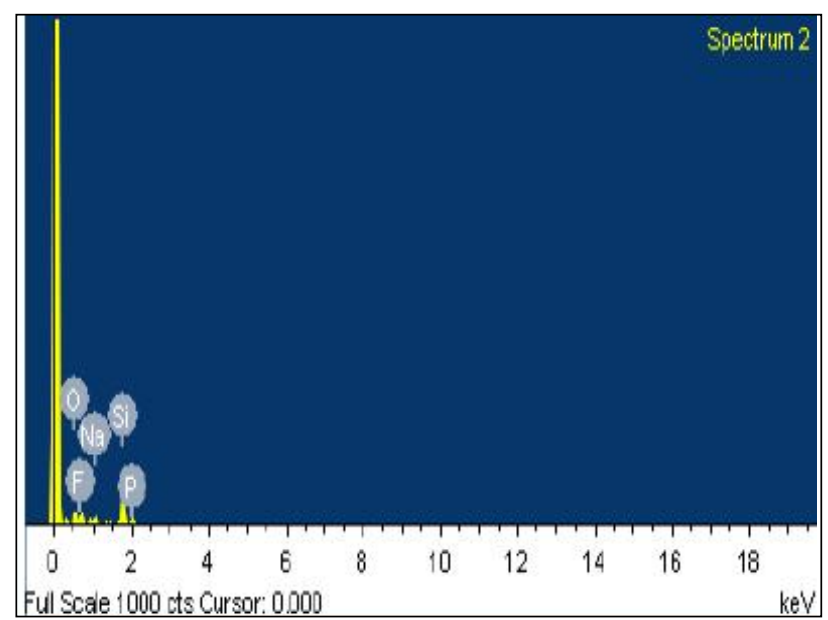

(b)

Figure 5. (a) SEM image and (b) EDS spectra of $\mathrm{Cu}$ electrolessly deposited on the sample whose surface is prepared through Route 1 in the $\mathrm{CH}_{3} \mathrm{COOH}$ bath.

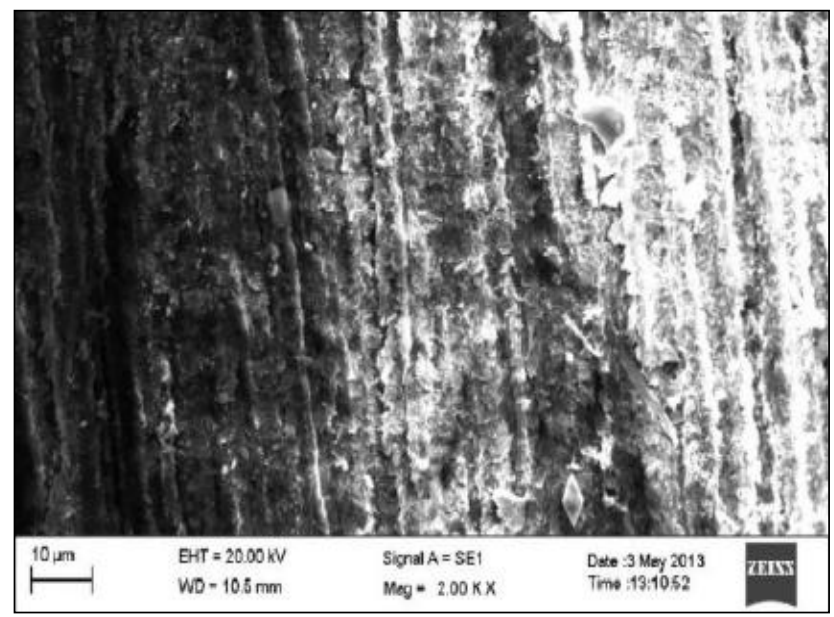

(a)

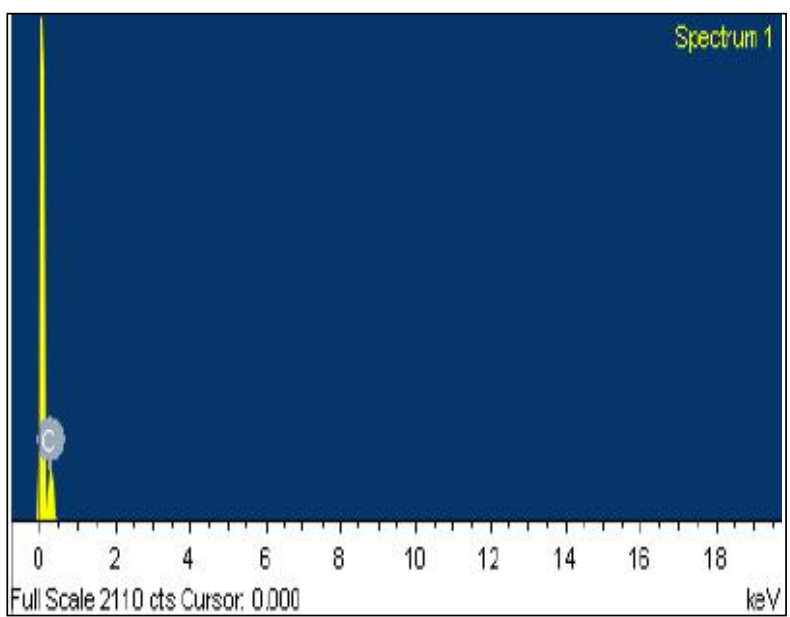

(b)

\subsection{SEM and EDS Characterization of Samples of Route 2}

Figure 6 corresponds to the SEM and EDS images obtained after depositing the samples in the HF bath. Small $\mathrm{Cu}$ particles dispersed in the part can be seen in the SEM image. The EDS spectra as shown indicate a small $\mathrm{Cu}$ peak at $8 \mathrm{keV}$, indicating $100 \% \mathrm{Cu}$, at the point where the spectra are taken. Figure 7 presents the results for the sample deposited in the $\mathrm{H}_{2} \mathrm{SO}_{4}$ bath. The SEM image is presented, having charged white areas where $\mathrm{Cu}$ deposition is expected. EDS spectra correspond to $94 \% \mathrm{Cu}$ and 
$6 \%$ C. Figure 8 depicts SEM and spectra for the sample deposited in the $\mathrm{H}_{3} \mathrm{PO}_{4}$ bath. The SEM image of the sample has a few $\mathrm{Cu}$ particles dispersed in the part. The EDS spectra correspond to $86 \% \mathrm{Cu}$, $11 \% \mathrm{O}$ and $3 \% \mathrm{P}$. Finally, the sample deposited in the $\mathrm{CH}_{3} \mathrm{COOH}$ bath is presented in Figure 9. The SEM image has a very poor appearance with no clear sign of $\mathrm{Cu}$ deposition. The elemental composition also shows no copper percentage.

For both of the routes, the distribution obtained is poor. As the $\mathrm{Cu}$ gets deposited only at the site where catalyst palladium (Pd) and/or tin ( $\mathrm{Sn}$ ) colloid has been deposited by removing butadiene from the ABS part during the etching stage of surface preparation. The etching may not be able to create cavities throughout the part, which leads to non-uniform or localized deposition of $\mathrm{Cu}$.

Figure 6. (a) SEM image and (b) EDS spectra of Cu electrolessly deposited on the sample whose surface is prepared through Route 2 in the HF bath.

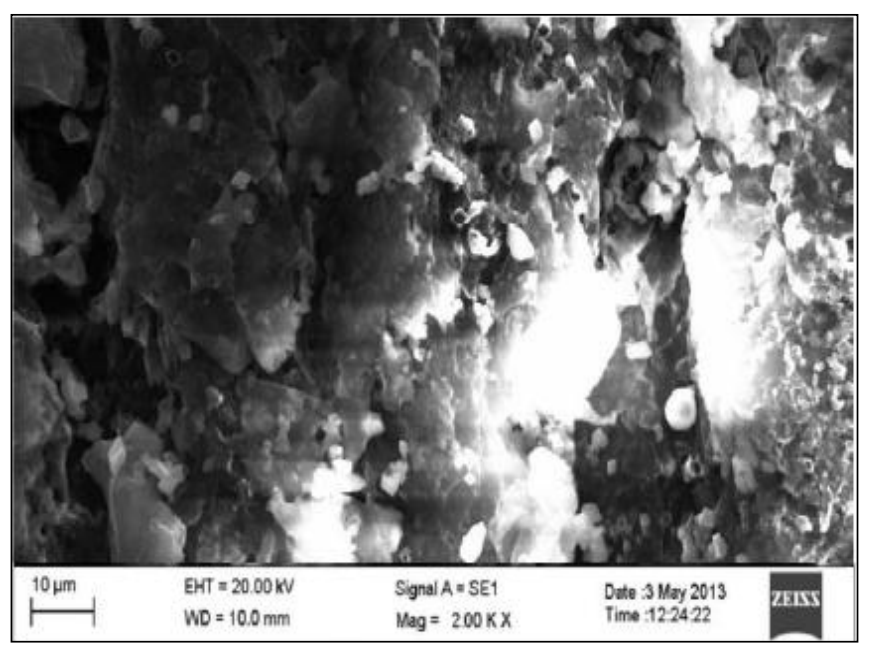

(a)

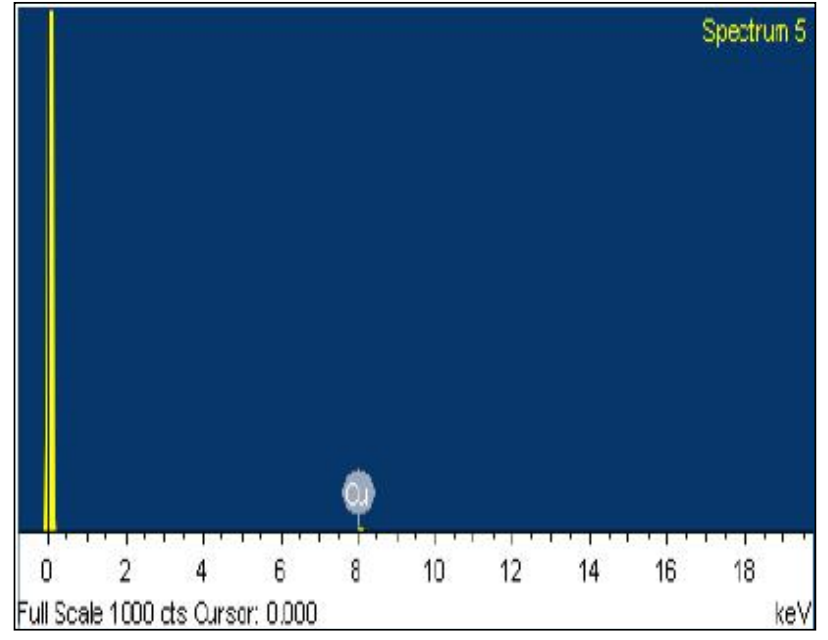

(b)

Figure 7. (a) SEM image and (b) EDS spectra of Cu electrolessly deposited on the sample whose surface is prepared through Route 2 in the $\mathrm{H}_{2} \mathrm{SO}_{4}$ bath.

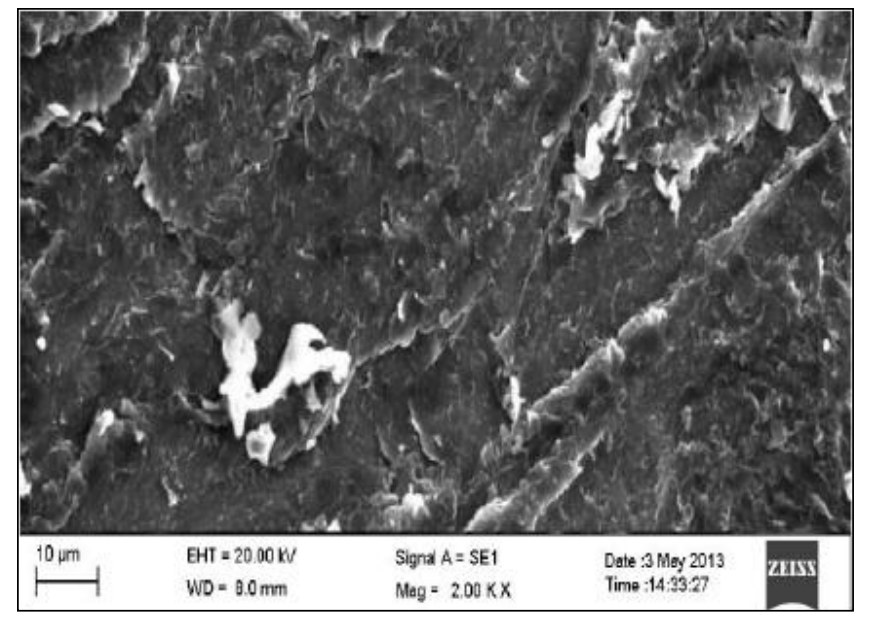

(a)

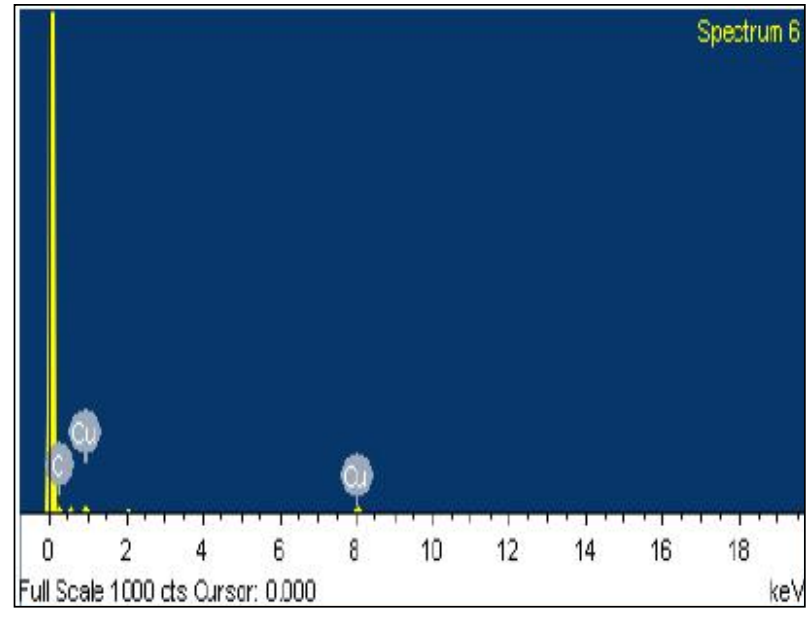

(b) 
Figure 8. (a) SEM image and (b) EDS spectra of Cu electrolessly deposited on the sample whose surface is prepared through Route 2 in the $\mathrm{H}_{3} \mathrm{PO}_{4}$ bath.

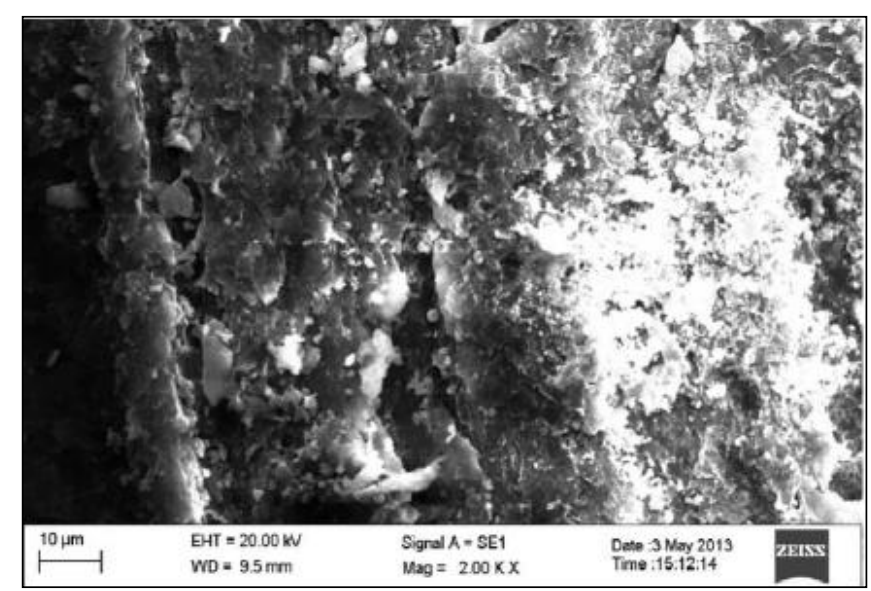

(a)

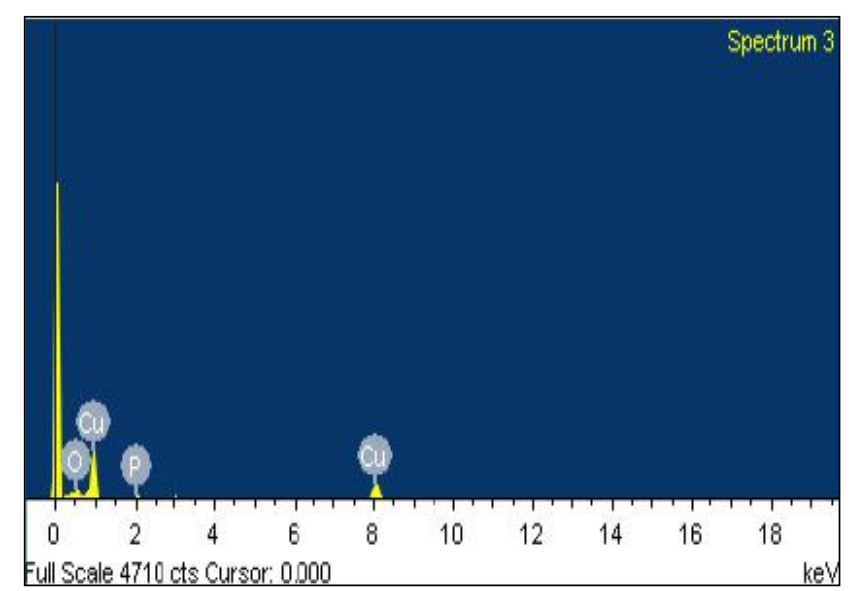

(b)

Figure 9. (a) SEM image and (b) EDS spectra of $\mathrm{Cu}$ electrolessly deposited on the sample whose surface is prepared through Route 2 in the $\mathrm{CH}_{3} \mathrm{COOH}$ bath.

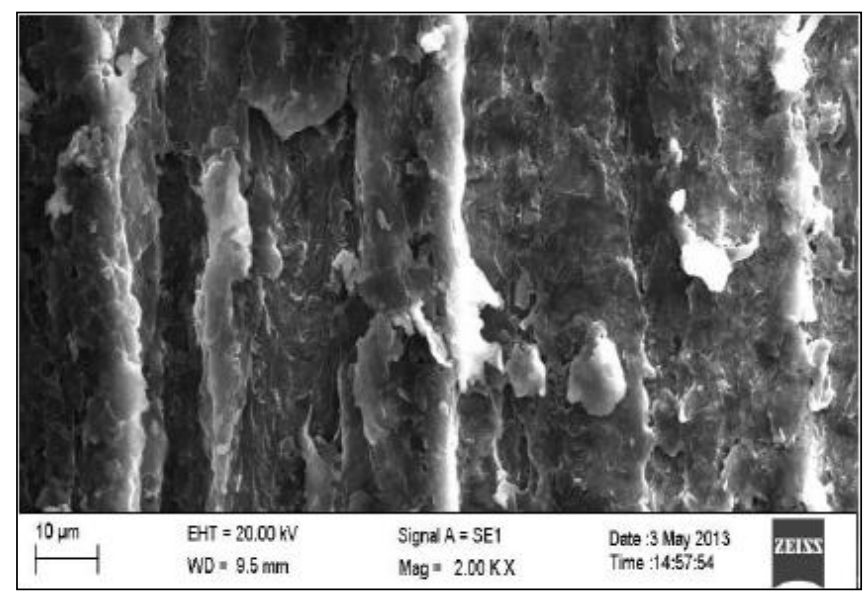

(a)

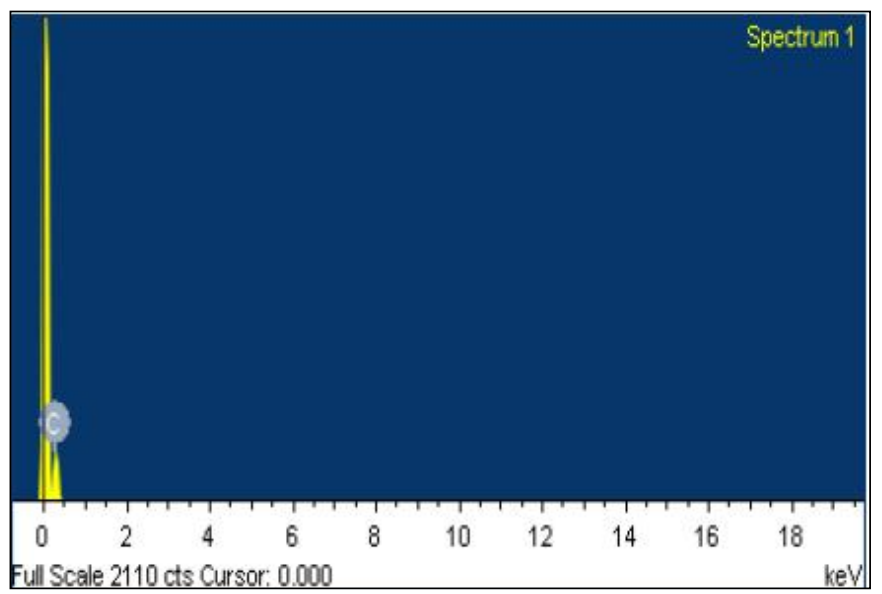

(b)

\subsection{Adhesion Evaluation for ABS Parts}

The adhesion evaluation of all of the ABS samples is done according to the scale defined by ASTM D 3359-02. All of the ABS test samples after the tape test show no flaking and yield results in a scale 4 or 5 , meaning excellent adhesion strength, as only less than $5 \%$ of the deposited materials could be removed by the tape (Table 3 ).

Table 3. Adhesion performance assessment of electroless deposited $\mathrm{Cu}$ layers on ABS parts.

\begin{tabular}{ccccc}
\hline \multirow{2}{*}{$\begin{array}{c}\text { Deposition } \\
\text { Condition }\end{array}$} & $\mathbf{H F}$ & $\mathbf{H}_{2} \mathbf{S O}_{4}$ & $\mathbf{H}_{3} \mathbf{P O}_{4}$ & $\mathbf{C H}_{3} \mathbf{C O O H}$ \\
\cline { 2 - 5 } & 5 & 5 & 5 & 4 \\
\hline Scale & 5 & 5 . & 5
\end{tabular}




\section{Conclusions}

Metallization of ABS parts has been studied on flat surfaces. Two different routes were employed for preparing the ABS surface for achieving the metallization on them. These routes applied are:

Route 1: ABS parts prepared using chromic acid for etching.

Route 2: ABS parts prepared using $\mathrm{H}_{2} \mathrm{SO}_{4} / \mathrm{H}_{2} \mathrm{O}_{2}$ for etching.

After surface preparation using these two routes, $\mathrm{Cu}$ is deposited electrolessly using four different acidic baths. The acidic baths used are $5 \mathrm{wt} \% \mathrm{CuSO}_{4}$ with $15 \mathrm{wt} \%$ of individual $\mathrm{HF}, \mathrm{H}_{2} \mathrm{SO}_{4}, \mathrm{H}_{3} \mathrm{PO}_{4}$ and $\mathrm{CH}_{3} \mathrm{COOH}$ acids. $\mathrm{Cu}$ deposition in different acidic baths for both routes was presented with their electrical performance measurements, SEM images and EDS analyses in this study.

A better deposition of copper is observed through Route 1 when compared. Although Route 2 provides a conductivity value in the three baths, except $\mathrm{CH}_{3} \mathrm{COOH}$, the sample prepared through Route 2 shows better conductivity in the $\mathrm{HF}$ and $\mathrm{H}_{2} \mathrm{SO}_{4}$ bath, though no conductivity in remaining acidic bath. It is also important to state that a varying amount of conductivity was obtained for different acidic baths used. The reason for the varying conductivity is due to the different size and non-uniform distribution of $\mathrm{Cu}$ in each route and in different acidic baths. Furthermore, an important observation is that the conductivity improves with the deposition time for the entire acidic bath showing conductivity. Furthermore, it can be observed that for both of the routes, the $\mathrm{H}_{2} \mathrm{SO}_{4}$ bath gives the best result with no conductivity for the $\mathrm{CH}_{3} \mathrm{COOH}$ bath. Hence, $\mathrm{H}_{2} \mathrm{SO}_{4}$ is the most effective acid used in this study. This is further validated with the SEM image and EDS spectra. Thus, it can be noted that the $\mathrm{H}_{2} \mathrm{SO}_{4}$ bath is the best suited for the electroless deposition of copper on ABS parts. Furthermore, from the above experimental work, it can be concluded that the $\mathrm{CH}_{3} \mathrm{COOH}$ bath is not suitable for electroless metallization.

Based on the current results, the development of a simpler and more cost-efficient $\mathrm{Cu}$ plating bath to further optimize the deposition conditions (the concentration of acids, deposition temperature and deposition time) is desired. Besides these, the lack of uniformity of $\mathrm{Cu}$ layers deposited under current conditions may become another focus of our future efforts.

\section{Acknowledgement}

All the experimental work is carried out in the Rapid Prototyping Lab of National Institute of foundry and forge technology and SEM/EDS images are taken at RDCIS, Ranchi. We also acknowledge the unanimous referees for their valuable comment in the preparation of this technical paper.

\section{Author Contributions}

The whole work is possible because of immense effort of both the authors Azhar equbal and Anoop kumar Sood. This work is very dedicatedly supervised by Anoop kumar Sood. The idea was taken from the literature but none of the literature has compared both the routes and the performance was not measured. Thus both the authors decided to compare them and tried to draw the conclusion. The individual idea was also implemented without confliction. Thus the work is made possible. 


\section{Conflicts of Interest}

The authors declare no conflict of interest.

\section{References}

1. Kuzmik, J.J.; Mallory, G.O.; Hajdu, J.B. Electroless Plating: Fundamentals and Applications; The American Electroplaters and Surface Finishers Society: Orlando, FL, USA, 1990.

2. Mittal, K.L. Metallized Plastics Fundamental and Applied Aspects, 1st ed.; VSP BV: Utrecht, The Netherlands, 2001.

3. Domenech, S.; Lima, E., Jr.; Drago, V.; Lima, J.; Borges, N.G., Jr.; Avila, A.; Soldi, V. Electroless plating of nickel-phosphorous on surface-modified poly(ethylene terephthalate) films. J. Appl. Surf. Sci. 2003, 220, 238-250.

4. Zhang, M.C.; Kang, E.T.; Neoh, K.G.; Tan, K.L. Electroless plating of copper and nickel on surface modified poly (tetrafluoroethylene) films. J. Electrochem. Soc. 2001, 148, 71-80.

5. Radulescu, F.; Miller, P.; Cunnane, L.; Harris, M.; Lam, H.; Bowers, C. Complete sputtering metallization for high-volume manufacturing. J. III Vs Rev. 2002, 15, 42-45.

6. Griehl, S.; Muller, T.; Winkler, R. Thick metallization-layers on polymers through vacuum-technology. J. Surf. Coat. Technol. 2003, 169, 24-26.

7. Long, P.; Blackburn, M.; Watkins, J. Chemical fluid deposition: A hybrid technique for low temperature metallization. J. Adv. Mater. 2000, 12, 913-915.

8. De Bruyn, K.; van Stappen, M.; de Deurwaerder, H.; Rouxhet, L.; Celis, J.P. Study of pretreatment methods for vacuum metallization of plastics. J. Surf. Coat. Technol. 2003, 163-164, 710-715.

9. Hanna, F.; Hamid, A.; Aal, A. Controlling factors affecting the stability and rate of electroless copper plating. J. Mater. Lett. 2003, 58, 104-109.

10. Nicolas, D.; Pascu, M.; Vasile, C.; Poncin, F. Influence of the polymer pre-treatment before its electroless metallization. J. Surf. Coat. Technol. 2006, 200, 4257-4265.

11. Wang, X.; Li, N.; Li, Y. Effect of Pd ions in the chemical etching solution. J. Univ. Sci. Technol. 2007, 14, 286-289.

12. Dia, L.; Liua, B.; Songb, J.; Shanb, D.; Yangb, D. Effect of chemical etching on the Cu/Ni metallization of poly (ether ether ketone)/carbon fiber composites. J. Appl. Surf. Sci. 2011, 257, 4272-4277.

13. Sood, A.K.; Ohdar, R.K.; Mahapatra, S.S. Improving dimensional accuracy of Fused Deposition Modeling processed part using grey Taguchi method. J. Mater. Design 2009, 30, 4243-4252.

14. Sood, A.K.; Ohdar, R.K.; Mahapatra, S.S. Parametric appraisal of mechanical property of fused deposition modeling processed parts. J. Mater. Design 2010, 31, 287-295.

15. Lustraflex Material Safety Data Sheet; Leathertone Plastic Supply: Findlay, OH, USA, 2009.

16. Charbonnier, M.; Romand, M. Polymer pretreatments for enhanced adhesion of metals deposited by the electroless process. Int. J. Adhes. Adhes. 2003, 23, 277-285.

17. Luan, B.; Yeung, M.; Wells, W.; Liu, X. Chemical surface preparation for metallization of stereolithography polymers. J. Appl. Surf. Sci. 2000, 156, 26-38. 
18. Wang, G.-X.; Li, N.; Hu, H.-L.; Yu, Y.-C. Process of direct copper plating on ABS plastics. J. Appl. Surf. Sci. 2006, 253, 480-484.

19. Teixeira, L.A.C.; Santini, M.C. Surface conditioning of ABS for metallization without the use chromium baths. J. Mater. Process. Technol. 2005, 170, 37-41.

20. Shu, Z.; Wang, X. Environment-friendly Palladium free surface activation technics for ABS Surface. J. Appl. Surf. Sci. 2012, 258, 5328-5331.

21. Fritz, N.; Koo, H.; Wilson, Z.; Uzunlar, E.; Wen, Z.; Yeow, X.; Allen, S.; Kohl, P. Electroless deposition of copper on organic and inorganic substrates using a $\mathrm{Sn} / \mathrm{Ag}$ catalyst. J. Electrochem. Soc. 2012, 159, 386-392.

22. Wu, X.; Sha, W. Surface morphology of electroless copper deposits using different reducing agents, synthesis and reactivity in inorganic metal-organic and nano-metal chemistry. Taylor Francis 2008, 38, 292-296.

23. Ono, S.; Naitoh, K.; Osaka, T. Initial propagation stage of direct copper plating on non-conducting substrates. J. Electrochem. Acta 1999, 44, 3697-3705.

24. Naruskevicius, L.; Tamasauskaite-Tamasiunaite, L.; Xieliene, A.; Jasulaitiene, V.A. Co-based surface activator for electroless copper deposition. J. Surf. Coat. Technol. 2012, 206, 2967-2971.

25. Kelly, J.; Mongey, K.; Gobil, Y.; Torres, J.; Kelly, P. Room temperature electroless plating copper seed layer process for damascene inter level metal structures. J. Microelectron. Eng. 2000, 50, 473-479.

26. Hong, S.; Shin, C.; Park, J. Palladium activation of $\mathrm{TaN}_{x}$ barrier films for autocatalytic electroless copper deposition. J. Electrochem. Soc. 2002, 149, 85-88.

27. ASTM D3359-09 Standard Test Methods for Measuring Adhesion by Tape Test; American Society for Testing and Materials: West Conshohocken, PA, USA, 2010.

(C) 2014 by the authors; licensee MDPI, Basel, Switzerland. This article is an open access article distributed under the terms and conditions of the Creative Commons Attribution license (http://creativecommons.org/licenses/by/3.0/). 